\title{
IMAGEM CORPORAL E AUTOESTIMA ENTRE PACIENTES COM OSTOMIAS INTESTINAIS
}

\section{Eduardo Viana Ricardo', Carolina Magalhães dos Santos ${ }^{2}$ \& Thaís Aparecida de Castro Palermo $^{2}$}

RICARDO, E.V.; SANTOS, C.M. \& PALERMO, T.A.C. Imagem corporal e autoestima entre pacientes com ostomias intestinais. Perspectivas Online: Biológicas \& Saúde. v. 8, n 28, p.71-80, 2018.

\section{RESUMO}

O objetivo deste estudo foi analisar a associação entre a imagem corporal e a autoestima de pacientes com ostomias intestinais. Trata-se de uma pesquisa quantitativa, descritiva e de corte transversal, realizada no hospital Oncobeda, no Município de Campos dos Goytacazes/RJ. A amostra foi composta por 30 indivíduos em tratamento ambulatorial. Para coleta de dados foram usados três instrumentos: um questionário geral estruturado, a escala de satisfação com a aparência e a escala de autoestima de Rosenberg que foram aplicados na modalidade entrevista. Os dados foram analisados no software SPSS, versão 24.0. Observou-se um predomínio de mulheres, idosas, casadas, com baixa eescolaridade, em algum emprego, com uma renda familiar per capita de 2 a 3 salários mínimos e residentes no município onde este estudo foi realizado. A maioria possuía alguma crença e destes, a maioria se denominava católico. Houve um predomínio de indivíduos com colostomia, de caráter definitivo e recentemente instalada. A principal causa para a instalação de um dispositivo coletor para a eliminação das fezes desses pacientes foi o Câncer. Evidenciou-se que a maioria dos participantes apresentou baixa satisfação com a imagem corporal $(53,3 \%)$ e alta autoestima $(83,3 \%)$. Essas variáveis apresentaram uma correlação forte $(\mathrm{p}=0,754)$ e significativa $(P=0,06)$.

Palavras-chave: Enfermagem Oncológica; Ostoma; Câncer Colorretal. 


\begin{abstract}
The objective of this study was to analyze the association between body image and selfesteem of patients with intestinal ostomies. This is a quantitative, descriptive and crosssectional study, carried out at the Oncobeda hospital, in the city of Campos dos Goytacazes / RJ. The sample consisted of 30 individuals with outpatient treatment. For data collection, three instruments were used: a structured general questionnaire, the satisfaction scale with the appearance and the scale of self-esteem of Rosenberg that were applied in the interview modality. Data were analyzed in SPSS software, version 24.0. It was observed a predominance of women, elderly, married, with low and schooling, in

some employment, with a per capita family income of 2 to 3 minimum salaries and residents in the municipality where this study was carried out. The majority had some belief and of these, the majority denominated Catholic. There was a predominance of individuals with colostomy, of definitive character and recently installed. The main cause for the installation of a collecting device for the elimination of these patients' feces was Cancer. It was evidenced that the majority of participants presented low satisfaction with body image $(53.3 \%)$ and high self-esteem $(83.3 \%)$. These variables presented a strong correlation $(\mathrm{p}=0.754)$ and significant $(\mathrm{P}=0.06)$.
\end{abstract}

Keywords: Nursing Oncology; Ostoma; Colorectal Cancer.

\footnotetext{
${ }^{1}$ Institutos Superiores de Ensino do CENSA -ISECENSA - Pesquisador do Laboratório Semiologia e Semiotécnica em Enfermagem - LASSEN - Rua Salvador Correa, 139, Centro, Campos dos Goytacazes, RJ, CEP: 28035-310, Brasil.

2 Pesquisadora do Laboratório de Estudos em Saúde Pública - LAESP/ISECENSA

(*)e-mail: enfeduardoviana@ hotmail.com

Data de recebimento: 22/11/2018 Aceito para publicação: 10/12/2018
} 


\section{INTRODUÇÃO}

A palavra estoma significa toda abertura cirúrgica de uma víscera oca ao meio externo, direta ou indiretamente para eliminações de secreções. Dependendo do seguimento exteriorizado são designados: traqueostomia, quando realizado na traquéia; gastrostomia, no estômago; jejunostomia e ileostomia no intestino delgado; colostomia, no intestino grosso e, urostomia, no sistema urinário (LLANOS, 2013).

A ostomia gera alterações dos sistemas corporais, desequilíbrio emocional, social e espiritual. No paciente hospitalizado são exacerbadas, pois ao serem questionados demonstram dificuldade em compreender a cirurgia realizada e suas consequiências no cotidiano. Doenças inflamatórias e neoplasia do intestino induzem milhares de pessoas a recorrem anualmente à extração desde órgão para manutenção da sobrevivência, porém o preconceito, mito e desinformação sobre a ostomia dificultam a reabilitação e socialização do paciente. É a mobilização temporária ou permanente de uma alça do cólon por meio de incisão no músculo reto anterior para expor o cólon transverso ou incisão músculo reto esquerdo para exposição do cólon sigmóide descendente. Esta cirurgia é realizada para tratar obstrução do sigmóide, decorrente de lesão maligna, inflamação avançada ou traumatismo que resultou em obstrução do cólon (TERIAKY, 2012).

Os estomas podem ser classificados em definitivo, devido à amputação completa do órgão sem haver solução de continuidade tornando o paciente incontinente fecal, após insucesso de outras ações que visam restaurar a evacuação transanal. Estomas temporários são usados em situações de trauma abdominal, com perfuração intestinal, mais comumente, por arma de fogo e perfuração por arma branca (HABR-GAMA e ARAÚJO, 2000).

As principais patologias que levam à colostomia são: a doença de Crohn, a megacólon chagásico, a doença diverticular dos cólons, a colite ulcerativa e o câncer colorretal. Dados do Instituto Nacional do Câncer (2016) indicam que os números de novos casos de cânceres de cólon e reto no Brasil no ano de 2016 foram 34.415, sendo 16.660 homens e 17.620 mulheres.

O paciente colostomizado pode envolver-se em situações difíceis como a inexperiência da equipe cirúrgica, o abandono da confecção do estoma e o regime de cirurgia. As complicações agudas são hemorragia, edema, infecção subcutânea, formação de necrose e fístula. As complicações tardias podem ser estenose, hérnia, retração e prolápso. O material fecal na pele e na região perístoma, causam reações e dermatites, que caracterizam-se por eritema, ulcerações ao redor da colostomia dentre outras alterações. (MANSOURZADEH et al., 2016).

Assim, as políticas de saúde e programas de saúde específicos, priorizam os direitos dos pacientes ostomizados, prestam um cuidado sistematizado, favorecendo o processo de reabilitação e reinserção social. A interdisciplinaridade entre profissionais das diversas áreas de saúde induzem o processo de reabilitação eficaz, favorecendo adaptação, qualidade de vida auto-estima e imagem corporal dos pacientes em questão (VARMA, 2011).

A contribuição efetiva e humana do enfermeiro na elaboração de plano assistencial é planejada durante todo o tratamento, incluindo sugestões de mudanças de rotina de horário, autocuidado, tipo de alimentação, higiene, manuseio com a bolsa, tipo de drenagem e prevenção contra complicações. O processo de educação permanente inicia-se na fase pré-operatória, momento 
de estabelecer vínculos com os pacientes e os familiares para que ambos possam entender a real situação e adaptarem-se à nova fase de vida (SANTOS, 2006).

Assim sendo, este estudo teve como objetivo analisar a associação entre a imagem corporal e a autoestima de pacientes com ostomias intestinais.

\section{METODOLOGIA}

Trata-se de uma pesquisa quantitativa, descritiva e de corte transversal, realizada no período de maio a novembro de 2017, no hospital Oncobeda, do Município de Campos dos Goytacazes/RJ. As informações da pesquisa foram coletadas através de três instrumentos: um questionário geral estruturado, a escala de satisfação com a aparência e a escala de autoestima de Rosenberg.

O questionário geral estruturado foi composto por dados sócio-demográficos (sexo, idade, estado civil, escolaridade, profissão, renda familiar, cidade onde mora e religião) e clínicos, relacionados à ostomia (tipo causa, tempo de estomização, e critério de permanência).

A Escala de Satisfação com a Aparência/ESA, corresponde ao Fator 1 da versão brasileira da Escala de Avaliação da Satisfação com a Imagem Corporal adaptada e validada no Brasil por Ferreira e Leite (2012). Esta escala não foi utilizada na íntegra, pois o Fator 2 aborda aspectos relativos a satisfação com o peso não sendo este o foco desta pesquisa. A ESA é composta por 18 itens relacionados ao grau de satisfação com a própria aparência, tanto nos aspectos intrínsecos, tidos como pessoais, quanto extrínsecos, refletidos em fotos e na visão dos outros.

A Escala de Autoestima de Rosenberg também foi adaptada e validada no Brasil (DINI et al., 2004). Ela é composta por 10 itens divididos em 6 enunciados positivos e 4 negativos, abordando sentimentos de autoaceitação e autodepreciação. Os intrumentos foram aplicados na modalidade entrevista.

A amostra obedeceu ao critério de conveniência, sendo incluídos os pacientes que frequentaram o hospital durante o período da coleta de dados; maiores de 18 anos e com estomas do tipo colostomia ou íleostomia e, excluídos os sujeitos com déficit cognitivo grave que os impossibilitava de responder as perguntas.

Para a análise dos dados, elaborou-se um banco de dados no software Statistical Package for the Social Science - SPSS, versão 24.0, onde os mesmos foram digitados e, posteriormente analisados. Para a análise da relação entre a imagem corporal e a autoestima dos indivíduos ostomizados, foi utilizado do Teste de Correlação de Spearman, sendo considerada como uma correlação fraca a variação do valor de $P$ entre 0,1 e 0,3 ; a moderada entre 0,4 e 0,6 ; a forte entre 0,7 e 0,9 e, a perfeita o valor de 1,0 , independente da positividade ou da negatividade dos valores (DANCEY e REIDY, 2013).

O presente projeto foi submetido ao Comitê de Ética em Pesquisa com Seres Humanos do ISECENSA (Campos dos Goytacazes/RJ), para apreciação ética. A coleta de dados foi realizada mediante a assinatura do Termo de Consentimento Livre e Esclarecido (TCLE) pelos participantes, atendendo às normas estabelecidas na Resolução 466/12 do Conselho Nacional de Saúde (BRASIL, 2012). 


\section{RESULTADOS}

Participaram deste estudo 30 pacientes em tratamento ambulatorial no hospital Oncobeda, no período de maio a novembro de 2017. Após as análises, observou-se uma amostra predominantemente feminina (60\%), com 61 anos ou mais $(46,7 \%)$, com estado civil caracterizado como casadas $(46,7 \%)$, com ensino fundamental $(56,7 \%)$, em algum emprego $(63,3 \%)$, percebendo uma renda familiar per capita de 2 a 3 salários mínimos (50\%) e residentes em Campos dos Goytacazes/RJ (56,7\%). Foi questionado sobre as crenças religiosas dos participantes e, 93,3\% disseram crer. Dos que crêem, 60,7\% se declaram católicos, conforme tabela 1 abaixo.

Tabela 1: Caracterização sociodemográfica dos sujeitos, Campos dos Goytacazes/RJ, 2017.

\begin{tabular}{|c|c|c|}
\hline \multirow[t]{2}{*}{ Variáveis } & \multicolumn{2}{|c|}{ Medidas Frequênciais } \\
\hline & n (30) & $\%$ \\
\hline \multicolumn{3}{|l|}{ Sexo } \\
\hline Feminino & 18 & 60,0 \\
\hline Masculino & 12 & 40,0 \\
\hline \multicolumn{3}{|l|}{ Faixa Etária } \\
\hline Até 49 anos & 9 & 30,0 \\
\hline De 50 a 60 anos & 7 & 23,3 \\
\hline 61 anos ou mais & 14 & 46,7 \\
\hline \multicolumn{3}{|l|}{ Estado Civil } \\
\hline Casado & 14 & 46,7 \\
\hline Divorciado & 5 & 16,7 \\
\hline Solteiro & 4 & 13,3 \\
\hline Viúvo & 7 & 23,3 \\
\hline \multicolumn{3}{|l|}{ Escolaridade } \\
\hline Fundamental & 17 & 56,7 \\
\hline Médio & 10 & 33,3 \\
\hline Superior & 3 & 10,0 \\
\hline \multicolumn{3}{|l|}{ Ocupação } \\
\hline Aposentado & 6 & 20,0 \\
\hline Do lar & 3 & 10,0 \\
\hline Doméstica & 2 & 6,7 \\
\hline Em algum emprego & 19 & 63,3 \\
\hline \multicolumn{3}{|l|}{ Renda Familiar per capita } \\
\hline Até 1 salário mínimo & 9 & 30,0 \\
\hline De 2 a 3 salários mínimos & 15 & 50,0 \\
\hline 4 ou mais salários mínimos & 4 & 13,3 \\
\hline Não responderam & 2 & 6,7 \\
\hline \multicolumn{3}{|l|}{ Zona de Residência } \\
\hline Campos dos Goytacazes & 17 & 56,7 \\
\hline
\end{tabular}


Outras cidades

\section{Religião}

Não creem

Creem

Tipo de religião dos que creem

Evagélicos

Católicos
13

2

28

11

17
43,3

6,7

93,3

39,3

60,7

Quanto à caracterização clínica dos sujeitos, 73,3\% possuíam estoma do tipo colostomia, $73,4 \%$ causado por Câncer, 73,3\% eram definitivos e o tempo médio de ostomia foi de 4,8 anos (DP=4,6 anos) de acordo com a tabela 2 abaixo.

Tabela 2: Caracterização clínica dos sujeitos, Campos dos Goytacazes/RJ, 2017.

\begin{tabular}{l|c|c}
\hline \multicolumn{1}{c|}{ Variáveis } & \multicolumn{2}{c}{ Medidas Frequênciais } \\
\hline \multicolumn{1}{c}{ Tipo de Estoma } & n (30) & \% \\
$\quad$ Colostomia & 22 & 73,3 \\
$\quad$ Iliostomia & 8 & 26,7 \\
Causas & & \\
$\quad$ Câncer & 22 & 73,4 \\
$\quad$ Demais causas clínicas & 3 & 10,0 \\
Causas traumáticas & 5 & 16,6 \\
Permanência & & \\
$\quad$ Definitivo & 22 & 73,3 \\
$\quad$ Temporário & 7 & 23,3 \\
$\quad$ Não sabem & 1 & 3,3 \\
Tempo ostomizado & & \\
$\quad$ Até 5 anos & 20 & 66,7 \\
$\quad$ Acima de 5 anos & 10 & 33,3 \\
\hline
\end{tabular}

Evidenciou-se que a maioria dos participantes apresentou baixa satisfação com a imagem corporal $(53,3 \%)$, entretanto, a maioria apresentou autoestima alta $(83,3 \%)$. Vide tabelas 3 a seguir. 
Tabela 3: Detalhamento dos Escores de Satisfação com a Imagem Corporal e de Autoestima em uma amostra de ostomizados, Campos dos Goytacazes/RJ, 2017.

\begin{tabular}{l|c|c}
\hline \multicolumn{1}{c|}{ Variáveis } & \multicolumn{2}{c}{ Medidas Frequênciais } \\
\hline \multicolumn{1}{c}{ Satisfação com a Imagem Corporal } & n (30) & \% \\
$\quad$ Baixa & & \\
Alta & 16 & 53,3 \\
Autoestima & 14 & 46,7 \\
$\quad$ Baixa & & \\
Alta & 5 & 16,7 \\
\hline
\end{tabular}

Para uma melhor interpretação destes resultados, foi realizado o Teste de Correlação de Spearman entre a satisfação com a imagem corporal e a autoestima. $O$ teste indicou uma correlação forte e significativa $(P=0,06)$, ou seja, os itens variaram de forma correlata. Vide tabela 4 abaixo.

Tabela 4: Correlação entre Satisfação com a Imagem corporal e Autoestima em Ostomizados, Campos dos Goytacazes/RJ, 2017.

\begin{tabular}{l|c|c|c|c}
\hline \multicolumn{1}{c|}{ Variáveis } & Média & Desvio Padrão & *p & $\boldsymbol{P}$ \\
\hline Satisfação com a Imagem Corporal & $\begin{array}{c}139,4 \\
33,5\end{array}$ & $\begin{array}{c}308,1 \\
4,7\end{array}$ & 0,754 & 0,06 \\
Autoestima & 3,06 \\
\hline
\end{tabular}

*Correlação de Spearman

\section{DISCUSSÃO}

Estudos realizados por Melloti et al. (2013) e por Salomé et al. (2014) com pacientes ostomizados encontraram resultados semelhantes, sendo a maioria mulheres idosas. O predomínio de mulheres pode ser explicado pelo fato de que em sua maioria as mulheres utilizam mais os serviços de saúde que os homens (LEVORATO et al., 2014).

Observou-se um predomínio de indivíduos casados e com baixa escolaridade (ensino fundamental). No estudo realizado por Sena (2015), os indivíduos investigados, em sua maioria, viviam com companheiro e possuíam ensino fundamental incompleto. Estes dados diferem dos encontrados por Pedrazzi et al. (2010), onde a maioria dos homens viviam com o cônjuge e as mulheres viviam sozinhas. Sobre a escolaridade, Almeida et al. (2015) também encontrou em seu estudo sobre a feminização da velhice, um predomínio de baixo nível escolar. Este resultado pode ser explicado pelos valores culturais e sociais adotados na primeira metade do século passado, onde o papel da mulher se limitava aos cuidados domésticos, dificultando o acesso à Educação (MEIRELES et al., 2007).

Apesar da baixa escolaridade, a maioria dos sujeitos deste estudo encontrava-se em algum emprego. Este resultado corrobora com o encontrado por Pedrazzi et al. (2010), onde a maioria dos domicílios era chefiado por idosos. Entretanto, difere do estudo de Sena (2015), onde a maioria era aposentado, pensionista ou beneficiário. Essa variação pode estar relacionada ao momento econômico e político em que o país se encontrava no período de realização da pesquisa. A baixa 
escolaridade pode justificar a renda familiar per capita encontrada, considerando que o mercado de trabalho tende a oferecer melhores salários aos mais capacitados.

A maioria dos indivíduos era residente de Campos dos Goytacazes/RJ, entretanto vale destacar que um quantitativo expressivo residia em outras cidades. Este resultado pode refletir a dificuldade de acesso às instituições de saúde por parte desses usuários em suas cidades de origem.

Menezes (2008) identificou altos índices de indivíduos que cultivavam alguma crença religiosa. Sena (2015) também encontrou uma maioria crente e de denominação católica. Estes corroboram com os resultados aqui evidenciados. O ser humano utiliza do recurso da fé para o alívio das suas dores, sendo este considerado um recurso de grande importância para tal finalidade. No cotidiano de pacientes ostomizados, a crença em alguma divindade é compreendida com uma estratégia de enfretamento (SALES et al., 2010).

Percebe-se um predomínio de colostomia, definitiva, recentemente instalada tendo como causa o Câncer. Resultados semelhantes foram encontrados no estudo de Sena (2015). Esta caracterização clínica é um reflexo da transição demográfica do país, resultando em novos perfis de morbimortalidade com altas prevalências das doenças crônico-degenartivas (INCA, 2014).

Os ostomizados em sua maioria possuíam uma baixa satisfação com a sua imagem corporal, embora apresentassem altos níveis de autoestima. Sena (2015) encontrou um resulatado próximo da paridade entre as categorias baixa e alta satisfação com a imagem corporal, porém com o mesmo comportamento encontrado no presente estudo para a autoestima. Embora a ideia de que, quanto maior a satisfação com a imagem corporal maior a autoestima, seja considerada verdadeira, outros fatores parecem contribuir para a autoestima destes indivíduos.

Em relação à imagem corporal, considera-se que a ostomia constitui a maior mudança na dimensão física do indivíduo, pois além de alterar a fisiologia normal de eliminação das fezes, causam inconvenientes como o odor e a utilização obrigatória do dispositivo coletor (SALES et al., 2010). Estes também enfrentam restrições e adaptações nos vestuários devido à nova condição (SUN et al., 2013).

No estudo de Sena (2015), os indivíduos melhoravam sua autoestima redirecionando suas preocupações para outras áreas de suas vidas capazes de lhe trazerem algum bem-estar. Esta foi identificada como uma forma de compensação diante das alterações na aparência impostas pela ostomia. Apesar de vários fatores influenciarem a satisfação com a imagem corporal, a autoestima parece estar relacionada ao momento de vida desses pacientes. A hipótese é de que o fato de já terem passado por todas as etapas da vida que antecedem a terceira idade, agregam valores a outros fatores que não a aparência física.

\section{CONCLUSÕES}

Ao final deste estudo observou-se um predomínio de mulheres, idosas, casadas, com baixa escolaridade, em algum emprego, com uma renda familiar per capita de 2 a 3 salários mínimos e residentes no município onde este estudo foi realizado. A maioria possuía alguma crença e destes, a maioria se denominava católico.

A maioria desses indivíduos possuía colostomia, de caráter definitivo e recentemente instalada. A principal causa para a instalação de um dispositivo coletor para a eliminação das fezes desses pacientes foi o Câncer. Apresentaram baixa satisfação com a imagem corporal e alta autoestima. A relação entre essas duas variáveis foi considerada forte e significativa.

Persp. online: biol. \& saúde, Campos dos Goytacazes, 28 (8),71-80, 2018

seer.perspectivasonline.com.br 
Apesar das alterações impostas pela nova condição, os pacientes ostomizados mantiveram alta autoestima. Considera-se que o apoio institucional e o apoio social são de grande importância para a qualidade de vida desses sujeitos. Várias atividades são preconizadas no sentindo de favorecer o enfretamento desta condição, como a consulta de enfermagem, o diálogo familiar e a troca de experiências entre os pacientes.

Vale ressaltar que o número amostral foi um fator limitante deste estudo, impossibilitando a extrapolação destes resultados para toda a população de ostomizados. Por isso, estudos futuros com uma amostra maior devem ser realizados.

\section{REFERÊNCIAS}

ALMEIDA, A.V.; MAFRA, S.C.T.; SILVA, E.P.; KANSO, S. A Feminização da Velhice: em foco as características socioeconômicas, pessoais e familiares das idosas e o risco social. Textos \& Contextos (Porto Alegre), v. 14, n. 1, p. 115 - 131, jan./jun. 2015. Disponível em $<$ http://www.scielo.com.br>. Acesso em 28 de junho de 2018.

BARBOSA, M.H.; DAL POGGETTO, M.T.; BARICHELlO, E.; CUNHA, D.F.; SILVA, R.; ALVES, P.I.C.; LUIZ, R.B. Aspectos Clínicos e Epidemiológicos de Estomizados Intestinais de um Município de Minas Gerais. REAS:3(1):64-73, 2014. Disponível em <http://www.scielo.com.br>. Acesso em 20 de fevereiro de 2017.

BRASIL. Conselho Nacional de Saúde. Resolução no 466/12, de 12 de dezembro de 2012. Aprova as diretrizes e normas regulamentadoras das pesquisas envolvendo seres humanos. Diário Oficial da União. Brasília/DF, 2012. Disponível em: <http://conselho.saude.gov.br/resolucoes/2012/reso466.pdf>. Acesso em 10 de fevereiro de 2017.

DANCEY, C.P.; REIDY, J. Estatística sem matemática para psicologia. Porto Alegre: Penso, 2013.

DINI, G.M.; QUARESMA, M.R.; FERRERIRA, L.M. Adaptação cultural e validação da versão brasileira da Escala de Autoestima de Rosenberg. Rev. Soc. Bras. Cir. Plást., 19(1), 41-52, 2004. Disponível em <http://www.rbcp.org.br>. Acesso em 20 de fevereiro de 2017.

FERREIRA, M.C.; LEITE, N.G.M. Adaptação e validação de um instrumento de avaliação de satisfação com a imagem corporal. Avaliação Psicológica. 1 (2), 141-149, 2012. Disponível em $<$ http://pepsic.bvsalud.org>. Acesso em 20 de fevereiro de 2017.

HABR-GAMA, A.; ARAÚJO, S.E.A. Estomas intestinais: aspectos conceituais e técnicos. In: Santos VLCG, Cesaretti IUR (Eds). Assistência em estomaterapia: cuidando do estomizado. São Paulo: Atheneu; 2000: 39-50.

INCA. Instituto Nacional de Câncer. Estimativa 2014: Incidência de Câncer no Brasil. Rio de Janeiro:2014. Dispnível em http://www.inca.gov.br. Acesso em 28 de junho de 2018.

INCA. Instituto Nacional de Câncer. Estimativa 2016: Incidência de Câncer no Brasil. Rio de Janeiro:2016. Dispnível em http://www.inca.gov.br. Acesso em 10 de fevereiro de 2017.

LEVORATO, C.D.; MELLO, L.M.; SILVA, A.S.; NUNES, A.A.. Fatores associados à procura por serviços de saúde numa perspectiva relacional de gênero. Ciênc. saúde coletiva [online], vol.19, n.4, 2014. Disponível em <http://www.scielo.com.br>. Acesso em 28 de junho de 2018.

LLANOS, E.B. Understanding the difficulty. Clin J Oncol Nurs: v. 17, n. 2: 2013.

MANSOURZADEH, A.R.; NIR, M.S.; MEHRI, S.N.; ZENDEHDELl, K. Living with malignant wound in cancer ptients and adolescents with non-cancer diagnosis: a meta-synthesis of 
qualitative studies. Int J Pediatr, v. 4, n. 9 (33): 3431-3440, 2016. Disponível em $<$ http://www.scielo.com.br>. Acesso em 20 de fevereiro de 2017.

MEIRELES, V.C.; MATSUDA, L.M.; COIMBRA, J.A.H.; MATHIAS, T.A.F. Características dos idosos em área de abrangência do Programa Saúde da Família na região noroeste do Paraná: contribuições para a gestão do cuidado em enfermagem. Revista Saúde e Sociedade, v. 16, nº 1 , 2007. Disponível em http://www.scielo.br. Acesso em 28 de junho de 2018.

MELLOTI, L.F.; BUENO, I.M.; SILVEIRA, G.V.; SILVA, M.E.N.; FEDOSSE, E. Characterizacion of patients with ostomy treated at a public municipal and regional reference center. J. Coloproctol: 33(2), 2013. Disponível em <http://www.scielo.com.br>. Acesso em 28 de junho de 2018.

MENEZES, M.M.P.N.C. Satisfação conjugal, auto-estima e imagem corporal em indivíduos ostomizados. [Dissertação de Mestrado]. Mestrado em Ciências da Educação. Universidade de Lisboa, 2008. Disponível em <http://repositorio.ul..pt>. Acesso em 28 de junho de 2018.

PEDRAZZI, E.C.; DELLA MOTTA, T.T.; VENDRÚSCOLO, T.R.P.; FABRÍCIO-WEHBE, S.C.C.; CRUZ, I.R.; RODRIGUES, R.A.P. Arranjo domiciliar dos idosos mais velhos. Rev. Latino-Am. Enfermagem: 18(01), 2010. Disponível em <http://www.scielo.com.br>. Acesso em 28 de junho de 2018.

SALES, C.A.; VIOLIN, M.R.; WAIDMAN, M.A.P.; MARCOM, S.S.; SILVA, M.AP. Sentimentos de pessoas ostomizadas: compreensão existencial. Rev. Esc. Enf. USP: 44(1), 221227, 2010. Disponível em <http://www.scielo.com.br>. Acesso em 28 de junho de 2018.

SALOMÉ, G.M.; ALMEIDA, S.A.; SILVEIRA, M.M. Quality of life and self-esteem of patients with intestinal stoma. Journal of Coloproctology: 34(4), 231-239, 2014. Disponível em $<$ http://www.sciencedirect.com>. Acesso em 28 de junho de 2018.

SANTOS, V.L.C.G. Cuidando do ostomizado: análise da trajetória no ensino, pesquisa e extensão (livre docência). Escola de Enfermagem da Universidade de São Paulo. São Paulo, p. 206, 2006.

SENA, R.M.C. Correlação entre imagem corporal e autoestima em pessoas com ostomias instetsinais. [Dissertação de Mestrado]. Universidade Federal do Rio Grande do Sul: 2015. Disponível em <http://www.scielo.com.br>. Acesso em 28 de junho de 2018.

SUN, V.; GRANT, M.; MCMULLEN, C.K.; ALTSCHULER, A.; MOHLER, M.J.; HOMNBROOK, M.C.; KROUSE, R.S. Surviving Colorectal Cancer: Long-Term, Persistent Ostomy-Specific Concerns and Adaptations. Journal of Wound, Ostomy, and Continence Nursing: Official Publication of The Wound, Ostomy and Continence Nurses Society/WOCN, 40(1), 61-72, 2013. Disponível em <http://www.scielo.com.br>. Acesso em 28 de junho de 2018.

TERIAKY, A.; GREGOR, J.; CHANDE, N. Percutaneous endoscopic gastrostomy tube placement for end estage palliation of malignant gastrointestinal obstructions. Saudi J Gastroenterol.: v. 18, n. 2:95-98, 2012.

VARMA, S. Second primary peristomal carcinoma: a case study. Br J Nurs.:v. 20, n. 16: S23-4, S26. 2011. 